\title{
Response latency and the content of immediate memory
}

\author{
PAUL FRAISSE and SERGE SMIRNOV \\ Laboratoire de Psychologie Expérimentale et Comparée Associé au C.N.R.S., Université René Descartes \\ et E.P.H.E., Se Section, 28, rue Serpente 75006 Paris, France
}

\begin{abstract}
We measured latency and speed of verbal responses to letters presented simultaneously (Experiment I) or in succession (Experiment II). In simultaneous presentation and within the limits of memory span, response latency increases proportionally to the number of.responses. In successive presentation, the same function is found when more than three responses are given. In both situations, response speed is constant. The increase in latency is about $70 \mathrm{msec}$ per response. Such a duration is too long to be explained by a process of encoding but too short to result from subvocal rehearsal. This latency seems to correspond to the preparation of the response.
\end{abstract}

The span of immediate memory has been investigated by many authors, but little attention has been given to variations of latency as a function of the number of items recalled.

The hypothesis tested here is that latency of the first response will increase with the number of items recalled. The aim of the present study is to examine the factors associated with this increase in latency. Is this increase due to processes of reception and processing of information or is it due to the preparation of the response?

In order to test the value of these two explanations, two situations which are classical in the study of immediate memory were compared. In one situation (Experiment I), stimuli were simultaneous and brief; in the second (Experiment II), they were presented successively. In both situations, the same type of response was required from the subject. The comparison of these two situations should enable us to clarify the respective role of the input and the output.

Furthermore, we measured the rate of responses, predicting that this index would throw some light on how information is processed.

\section{EXPERIMENT I}

\section{Response Latency to Letters Presented Simultaneously}

We measured the latency of the first response to a brief presentation of one to five letters.

Materials. The material consisted of the letters of the alphabet, with the exception of G, I, N, P, Q and W, which were omitted to avoid visual confusion. Black letters ( $4 \mathrm{~mm}$ high and $3 \mathrm{~mm}$ wide) were printed on white cards. Two spatial arrangements of letters and two types of responses resulted in the following two situations: Situation $A$-free recall: The letters were arranged in

Requests for reprints should be sent to P. Fraisse, Laboratoire de Psychologie Expérimentale et Comparée, 28, Rue Serpente, 75006-Paris, France. S. Smirnov is now at the University of Moscow, Faculty of Psychology, 18, Avenue Marx, Bât. 5, Moscow, USSR. random order on a circular surface (diameter, $28 \mathrm{~mm}$; visual angle, $1.3 \mathrm{deg}$ ) and the subjects were asked to recall the letters without regard to order. Situation $B-$ serial recall: The letters were arranged on a horizontal line, the space between two consecutive letters being $3 \mathrm{~mm}$. Subjects were asked to recall the letters in order, from left to right.

The number of letters printed on each card varied from one to five. For each number of letters and for each of Situations A and B, six different cards were constructed by randomly assigning letters to each card, so that an equally frequent presentation of all letters was obtained.

\section{Method}

Procedure. The cards were presented through a three-channel tachistoscope (Scientific Prototype, Model GB) during $50 \mathrm{msec}$, a duration that Sperling (1967) judged sufficient for identifying five letters. Luminance was $10 \mathrm{fL}$.

Before presenting the experimental material to the subject, the experimenter explained the two possible spatial arrangements and the two kinds of response, either free or serial recall. Subjects were asked to respond as quickly as possible and were given 16 practice cards (eight with letters in random order, eight in serial order).

All subjects were tested in Situations $A$ and $B$ (five in the order ABAB, five in the order BABA). In a given situation (A or $B)$, there were two presentations of the six sets of cards. The order of presentation of the cards was randomized in both situations. For each set of letters and for each situation, each subject gave 12 responses.

Subjects. Ten students in psychology, aged 19 to 25 years, served as subjects.

Recording of responses. Verbal responses were tape recorded, and a graphic recording was also made using a voice key connected to a polygraph on which the onset of the stimulus was also recorded. Thus, the latency of the first response and the successive intervals between responses from the onset of a response to the onset of the next response were recorded.

Due to malfunctioning of the polygraph, some measures (less than $4 \%$ ) were not recorded.

\section{Results}

The following analysis is based on correct responses, 


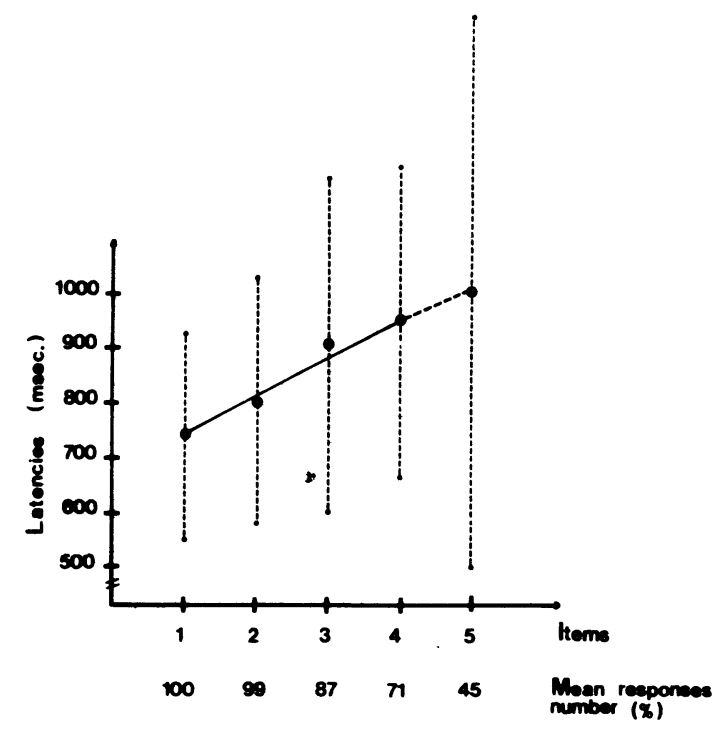

Figure 1. Time latency of the onset of the exact responses in the cases where the number of items in the response is equal to the number of items in the stimulus presented in simultaneity. The percentage of such cases is given on the line below the abscissa. The vertical dotted lines represent the variability $( \pm 1 \sigma)$.

i.e., responses in which all the elements from the stimulus were recalled.

(1) First response latency (TL). A comparison of the number of correct responses and the latencies in Situations A and B showed no significant difference between the two situations, and consequently the results were pooled. This lack of difference between the two situations is probably due to the fact that in this experiment we are within the limits of the memory span. Figure 1 shows that latency increased proportionally to the size of the content of immediate memory. For up to four letters (the limit of memory span) $\mathrm{TL}_{(\mathrm{msec})}=672+70 \mathrm{n}$, where $\mathrm{n}$ is the number of letters recalled.

(2) Rate of response. Analysis of variance revealed that the interresponse intervals in Situations A and B did not differ significantly.

Table 1 presents interresponse intervals for responses in which the number of letters is equal to the number of letters in the stimulus. Interresponse intervals did not differ significantly when the number of letters in the stimulus did not exceed the capacity of the memory span (four letters). These mean interresponse intervals were slightly shorter $(p<.05)$ than with five letters. With more than four letters, the variance of responses

Table 1

Interresponse Intervals (Milliseconds)

\begin{tabular}{ccccc}
\hline & \multicolumn{4}{c}{ Number of Letters in the Response } \\
\cline { 2 - 5 } Interval & 2 & 3 & 4 & 5 \\
\hline $1-2$ & 479 & 480 & 459 & 475 \\
$2-3$ & & 453 & 445 & 497 \\
$3-4$ & & & 426 & 536 \\
$4-5$ & & & & 490 \\
\hline
\end{tabular}

increased noticeably and the strategies used by the subjects were probably more diversified.

\section{EXPERIMENT II}

\section{Response Latency to Letters Presented Successively}

In this experiment, letters were presented in succession, and the presentation rate was low enough to permit the perception of a letter before the next letter was presented.

Materials. The letters of the alphabet (with the exception of $M, X$, and $W$ ) were used and projected on the same place. They were filmed in black and white and the film was projected on a screen at a distance of $3 \mathrm{~m}$ from the subject. The size of projected letters was $18 \mathrm{~mm}$. Each letter was presented for $600 \mathrm{msec}$, and there was a 300 -msec interval between two successive letters. After the appearance of the last letter, a star signaled the subject that he had to recall as quickly as possible the letters he had seen.

Eight different sets of sequences of letters were constructed, consisting of from one to eight randomly chosen letters, with an equally frequent presentation. Within each set, the order of the sequence was different and randomized. Two more sets of sequences were used for the practice session.

\section{Method}

Subjects. Two groups of 10 psychology students, aged 19 to 25 years, were tested individually.

Procedure. Different instructions were given to the two groups of subjects. Subjects in Group 1 were instructed to recall the letters in their serial position, whereas subjects in Group 2 were asked to recall the letters regardless of order. All subjects were asked to respond as fast as possible. After having seen two practice sequences, each subject saw the eight sets of sequences twice. The end of the presentation of each set was marked on the polygraph. A tape recorder and a voice key were used, as in Experiment $I$.

\section{Results}

The mean of the responses of both sets was computed, i.e., the mean of 16 responses per sequence length. The statistical analysis was based on 160 measures $(10 \times 16)$. As in Experiment I, the voice key failed to function in some cases and approximately $4 \%$ of the observations are missing.

(1) First response latency. Subjects in Group 2, who were instructed to recall the letters regardless of order, nevertheless tended to follow the order of presentation to the same degree as subjects in Group 1. Our criterion for an error was whether the response was displaced by more than one position from the order of presentation. (For instance, if a letter with rank 4 in the sequence stimulus was not recalled with rank 3,4 , or 5 , the response was scored as an error). There were $4.3 \%$ errors in Group 1 and $5.9 \%$ in Group 2. This difference was not significant.

Since there were no significant differences between Groups 1 and 2, the results were pooled.

Figure 2 presents the results for cases in which the 


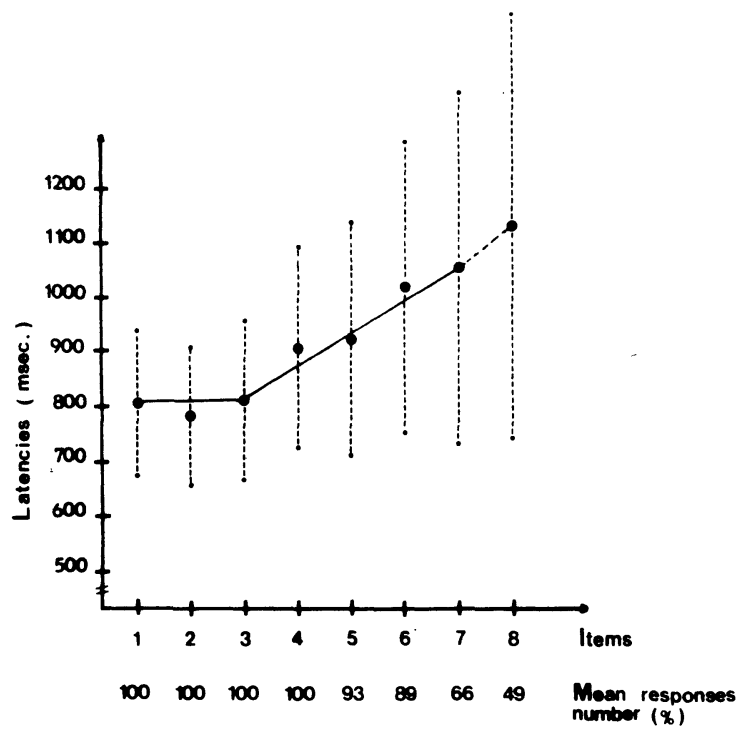

Figure 2. Mean response latency in the cases where the number of items in the response is equal to the number of items in the stimulus presented in succession. The percentage of such responses is given on the line below the abcissa. The vertical dotted lines represent the variability $( \pm 1 \sigma)$.

responses corresponded exactly to the stimuli. From one to three letters, the latency did not vary $(800 \mathrm{msec})$. From four to seven letters, the latency increased proportionally to the number of elements, with a mean increase of $67 \mathrm{msec} /$ letter. With eight letters, we exceeded the limits of memory span.

(2) Rate of response. We measured interresponse intervals without taking into account errors of position. The two groups differed markedly as far as speed of responses was concerned.

In Figure 3 the interval durations are plotted against their position within the series of responses, without taking response length into consideration.

In the serial order condition, the interresponse intervals were nearly constant (mean $=465 \mathrm{msec}$ ), whereas in free recall they were longer (mean $=616 \mathrm{msec}$ ) and increased regularly with the number of items recalled. Two stages may be distinguished: The first one is from one to three letters, and the other from four to eight letters. Between four and eight letters, there was a significant interaction between the magnitude of the intervals and the type of response.

\section{DISCUSSION}

Our findings quite clearly indicate that, within the limits of memory span, response latency for simultaneous presentation increases proportionally to the number of elements presented and recalled; the same function is found when more than three letters are presented in succession. When the response begins, the items to be recalled are all available, since the speed of responses is constant. This speed is slightly slower than the speed for enunciating the letters that are read $[475 \mathrm{msec}$ in Experiment I and $465 \mathrm{msec}$ in Experiment II. For reading, Mackworth (1963) found a speed of $333 \mathrm{msec}$ ].
Why is there an increase in latency with the number of stimuli?

According to Sperling (1967), this result cannot be explained by the amount of time for processing information in the VIS. This process would account for an increase of only $10 \mathrm{msec} / \mathrm{let}-$ ter when stimuli are presented tachistoscopically. Furthermore this argument is not relevant for Experiment II.

Would a subvocal rehearsal take place before enunciation? Subjects are not conscious of this phenomenon; furthermore, subvocal rehearsal is nearly as slow as vocal rehearsal [from 3 to 6 letters/sec (Landauer, 1962)]. Thus, subvocal rehearsal cannot explain an increase in latency of $67-70 \mathrm{msec}$ only per each item.

It is true that Eriksen, Pollack, and Montague (1970) and Klapp $(1971,1973)$ have shown that an implicit speech mechanism played a role in perceptual encoding. They have found that response latency to a word or to a two-digit number increased with the number of syllables in the stimulus: $11 \mathrm{msec} / \mathrm{syllable}$ according to Eriksen, $20 \mathrm{msec}$ according to Klapp. Klapp agrees that this duration is too short to be explained by rehearsal, and he suggests that an implicit representation is implied in the perception of words and of numbers. This process could intervene with a series of letters, but its duration is shorter than the one we have found and it cannot intervene when letters are presented in succession.

Our conclusion, then, is that the increase in response latency with the number of items is too slow to result from the encoding process and too fast to result from subvocal rehearsal in Experiment I. On the other hand, the encoding process cannot be involved in Experiment II, since the subject has the time to encode each letter and even to rehearse it.

As a consequence, we consider that the increase in latency with the number of responses is due to the preparation of the response.

However, we need an explanation for why the response latency in successive presentation does not increase when we pass from one to three letters. One may hypothesize that, with a presentation duration of $900 \mathrm{msec}$, up to three elements, the subject has the time to code the elements of the stimulus and prepare his answer. With more than three stimuli, interference between stimuli and competition between responses necessitate a preparation process of the response analogous to the one that occurs in simultaneous presentation.

A partial explanation of our results might be found at the level of response processes rather than at the level of coding and storage processes. In any case, since he utters all letters at the same speed, we know that when the subject begins to formulate his answer, his responses are available, except perhaps in the free recall condition with successive presentation. It seems that this
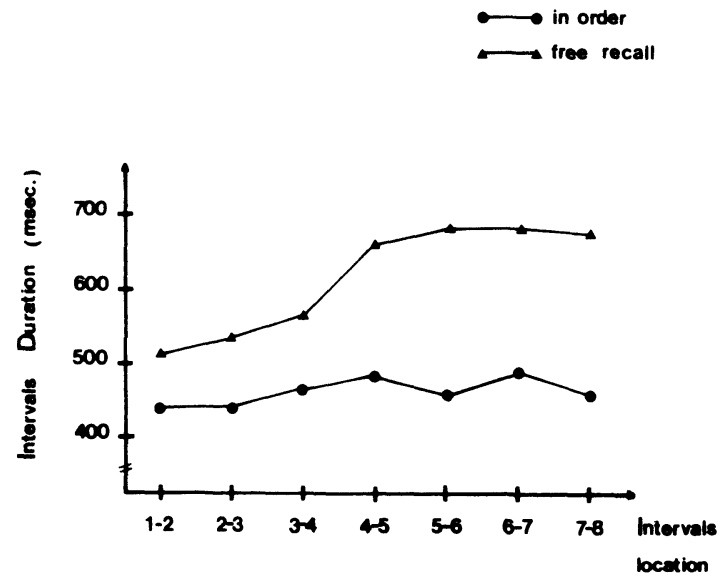

Figure 3. Mean duration of intervals as a function of their location within the sequence of responses. 
condition induces a hesitation concerning the choice of the order of response, even though all responses are available. Murdock and Okada (1970) have also found in the free recall of lists of 20 words that the rate of recall is inversely related to the number of available words.

A further experiment should test whether the increase in latency depends on the number of responses only, whatever the number of stimuli. But in the two experiments reported here, we have found that the results are very similar when latency is plotted against the number of letters recalled, whatever the number of letters presented.

\section{REFERENCES}

Eriksen, C. W., Pollack, M. D., \& Montague, W. E. Implicit speech: Mechanism in perceptual encoding. Journal of Experimental Psychology, 1970, 84, 502.

KLAPP, S. T. Implicit speech inferred from response latencies in same-different decisions. Journal of Experimental Psychology, 1971, 91, 262-267.
Klapp, S. T., Anderson, W. G., \& Berrian, R. W. Implicit speech in reading reconsidered. Journal of Experimental Psychology, 1973, 100, 368-374.

LANDAuer, T. K. Rate of implicit speech. Perceptual and Motor Skills, 1962, 15, 646.

MACKWORTH, J. F. The relation between the visual image and post-perceptual immediate memory. Journal of Verbal Learning and Verbal Behavior, 1963, 2, 75-85.

MURDOCK, B. B., \& OKADA, R. Inter-response times in single trial free recall. Journal of Experimental Psychology, 1970 , 86, 263-267.

SPERling, G. Successive approximations to a model for short term memory. Acta Psychologica, 1967, 27, 285-292.

(Received for publication April 23, 1976; revision accepted June 26,1976 .) 